\title{
Clinical Efficacy of Metoprolol Succinic Acid Sustained Release Tablets Combined with Trimetazidine in the Treatment of Gastrointestinal Tumors with Angina Pectoris
}

Shuo Huang, Yue Zhang, Jijun Li*

Jilin City Hospital of Chemical Industry, Jilin 132021, China

\begin{abstract}
Objective: To investigate the clinical efficacy of metoprolol succinate sustained-release tablets combined with trimetazidine in the treatment of gastrointestinal tumors with angina pectoris. Methods: We enrolled the 58 patients with digestive tract tumor merger angina in November 2017 - October 2019 and analysis the hospital clinical data by retrospective method. We included patients with routine treatment in control group ( $\mathrm{n}=31$ cases $)$ and the subjects treated with increased dose of succinic acid metoprololzyban joint with trimetazidineinobservation group $(n=27$ cases) according to the different treatment group. Results: The effective rate of angina pectoris treatment in the observation group was higher than that in the control group. Furthermore, the incidence of adverse reactions was lower than that in the control group and the difference was statistically significant $(P<0.05)$. Conclusion: Metoprolol succinic acid sustained release tablets combined with trimetazidine in the treatment of gastrointestinal tumors with angina pectoris can improve the efficacy of angina pectoris. The drug use is safe and worthy of clinical use.
\end{abstract}

Keywords: Angina; Gastrointestinal tumors; Sustained release metoprolol succinate; Trimetazidine

Publication date: March, 2020

Publication online: 31 March, 2020

*Corresponding author: Jijun Li, huangshuo0511@126. com

\section{Introduction}

The human digestive tract includes esophagus, stomach, small intestine, large intestine, liver, gallbladder, pancreas and other organs. Angina pectoris is a clinical syndrome characterized by acute myocardial ischemia and hypoxia caused by insufficient blood supply to coronary arteries. Angina is the heart ischemia reflections to the surface of a body feel pain which characteristic for chest paroxysmal, crushing pain and might be accompanied by other symptoms. The position of the pain is mainly in breastbone hind and can radiation to the area before the heart and left upper limb. It is cured by the drugspriority in clinical treatment such as succinic acid metoprololzyban and trimetazidine. Most of the patients can alleviate or disappearafter treatment the symptoms ${ }^{[1]}$. Based on thisreaearch, the clinical efficacy of metoprolol succinate sustained release tablets combined with trimetazidine in the treatment of gastrointestinal tumors with angina pectoris was analyzed as follows.

\section{Materials and methods}

\subsection{The general information}

The anthors enrolled the 58 patients with digestive tract tumor merger anginain November 2017 - October 2019 and analysis the hospital clinical data by retrospective method. We included patients with routine treatment in control group $(\mathrm{n}=31$ cases $)$ and the subjects treated with increased dose of succinic acid metoprololzyban joint with trimetazidineinobservation group $(n=27$ cases) according to the different treatment group. The control group included 23 males and 8 females. The age ranged from 41 to 75 years old, with an average of 
$(63.58 \pm 4.27)$ years old. The course of disease was $1-5$ years, with an average of $(3.49 \pm 1.08)$ years. Angina pectoris severity: II level 19 cases, III level in 11 cases, 1 case IV level. The observation group included 21 males and 6 females. The age ranged from 40 to 72 years old, with an average of $(63.54 \pm 4.32)$ years old. The course of disease ranged from 1 to 6 years, with an average of $3.51 \pm 1.06$ years. Angina pectoris severity: II level 15 cases, 10 cases III, IV level in 2 cases. The data of the two groups were compared, and the difference was not statistically significant $(P>0.05)$.

\subsection{Inclusion criteria and exclusion criteria}

(1) inclusion criteria: No history of allergy to the treatment drugs; Can be better with the completion of treatment; No major mental disorders. (2) exclusion criteria: Patients with severe abnormal liver and kidney functions; Merger of other serious cardiovascular and cerebrovascular diseases; Merger of other respiratory system, blood system, immune system diseases; there is a communication cognitive disorder.

\subsection{Methods}

Digestive tract tumors were diagnosed in both groups. Patients with symptomatic treatment were treated in combination with their own conditions, and those with indications of surgery were treated with surgery, followed by postoperative radiotherapy, chemotherapy and other comprehensive treatment.

\subsubsection{Control group}

Conventional treatment of angina pectoris in the control group. According to the symptomatic treatment of patients' own conditions, they were given rest in bed. Nitrate (nitroglycerin preparation) was used to dilate coronary arteries reducing resistance and increasing blood flow. The receptor blockers blocked the stimulation of sympathetic amines on heart rate and systolic force receptors. Calcium channel blockers inhibit myocardial contraction; reduce myocardial oxygen consumption, etc., treatment for 3 months.

\subsubsection{Observation group}

Metoprolol succinic acid sustained release tablets were administered on the basis of the control group (AstraZeneca AB, J20150045 approved by the state and specification: $95 \mathrm{mg}$ ) oral treatment, 1 piece/times, 1 / d, trimetazidine (pharmaceutical factory, jiangsuwuzhong pharmaceutical group co., LTD. Suzhou H20073709 approved by the state and specifications: $20 \mathrm{mg}$ ) treatment, 1 piece/time, 3 times/d, treatment for 3 months.

\subsection{Evaluation index}

(1) After 3 months of treatment, the efficacy of angina pectoris was compared between the two groups showing that the use of nitrate esters and the number of angina pectoris attacks were reduced by $>80 \%$. Effective: the use of nitrate esters and the number of angina attacks were reduced by $50 \%-80 \%$. Inefficacy: the use of nitrate and the number of angina attacks were reduced by $<50 \%$ or worse than before treatment. Total efficiency $=$ apparent efficiency + efficient.

(2) The incidence of adverse reactions, including nausea and vomiting, low blood pressure, dizziness and abdominal pain, was counted during the treatment period of the two groups.

\subsection{Statistical methods}

SPSS 22.0 software was used for data processing, and the counting data was expressed as a percentage.

\section{Results}

\subsection{Effect of angina}

The effective rate of angina pectoris treatment in the observation group was higher than that in the control group, and the difference was statistically significant $(P$ $<0.05)$. See Table 1.

Table 1. Comparison of the efficacy of angina pectoris between the two groups $n(\%)$

\begin{tabular}{ccccc}
\hline group & effect & valid & invalid & effective rate \\
\hline The control group $(\mathrm{n}=31)$ & $5(16.13)$ & $15(48.39)$ & $11(35.48)$ & $20(64.52)$ \\
Observation group $(\mathrm{n}=27)$ & $15(55.56)$ & $11(40.74)$ & $1(3.70)$ & $26(96.30)$ \\
$\mathrm{Z} / \chi^{2}$ & & $\mathrm{Z}=3.664$ & $\chi 2=8.882$ \\
$P$ & & 0.000 & 0.003 \\
\hline
\end{tabular}




\subsection{Adverse reaction}

The incidence of adverse reactions in the observation group was lower than that in the control group, and the difference was statistically significant $(P<0.05)$. See Table 2.

Table 2. Comparison of adverse reactions between the two groups n(\%)

\begin{tabular}{cccccc}
\hline Group & nausea and vomiting & hypotension & dizzy & abdominal pain & Always happen \\
\hline The control group $(\mathrm{n}=31)$ & $9(29.03)$ & $4(12.90)$ & $3(9.68)$ & $2(6.45)$ & $18(58.06)$ \\
Observation group $(\mathrm{n}=27)$ & $1(3.70)$ & $1(3.70)$ & $1(3.70)$ & $1(3.70)$ & $4(14.81)$ \\
$\chi^{2}$ & - & - & - & - & 11.466 \\
$P$ & - & - & - & - & 0.001 \\
\hline
\end{tabular}

\section{Discussion}

The direct cause of angina pectoris the absolute or relative shortage of myocardial blood supply. Therefore, many various factors which reduce myocardial blood (blood oxygen) supply and increase oxygen consumption inducing angina pectoris. Insufficient blood supply to the heart muscle is mainly due to coronary heart disease. Other types of heart disease or uncontrolled high blood pressure can also cause angina. Most of the patients were presented with dull pain, crushing pain or a sense of constriction behind the sternum and in the throat. Some patients had only chest tightness, which could be divided into typical angina pectoris and atypical angina pectoris.

The results of this study have shown that the effective rate of angina pectoris treatment in the observation group was higher than that in the control group. The incidence of adverse reactions was lower than that in the control group, indicating that metoprolol succinic acid sustained release tablets combined with trimetazidine in the treatment of gastrointestinal tumors with angina pectoris could improve the efficacy of angina pectoris in patients with safe medication. In the conventional treatment of angina pectoris, the functions of nitrate preparations are dilating coronary arteries, reducing resistance, increasing blood flow. However, dilating peripheral blood vessels, reducing the amount of venous return to the heart, reducing ventricular volume, intracardiac pressure, improving cardiac output and blood pressure and reducing the pre- and post-load of the heart and cardiac oxygen to relieve angina pectoris. Beta blockers have proposed sympathetic amine on the heart rate and contraction force the stimulating effect of the receptor. Moreover, slowing heart rate, lowering blood pressure, reducing the myocardial contraction force and oxygening consumption are the functions of
Beta blockers to relieve angina attack. While, the Beta blockers could also reduce the blood flow dynamic response to movement and reduce the myocardial oxygen consumption in the same physical activity levels not ischemic myocardium area small arteries narrow so that more blood could go through the extreme expansion of collateral circulation into the ischemic area. Calcium channel blockers can inhibit calcium ions from entering cells and also inhibit the utilization of calcium ions in excitation-contraction coupling of cardiomyocytes so as to inhibit myocardial contraction and reduce myocardial oxygen consumption. Dilating coronary artery, relieving coronary artery spasm, improving blood supply of myocardium under endocardium, dilating peripheral blood vessels, reducing arterial blood pressure, reducing cardiac load, reducing blood viscosity, anti-platelet aggregation and improve cardiac microcirculation are the function of the Calcium channel blockers.

Metoprolol succinic acid sustained release tablets consist of microencapsulated particles of metoprolol succinic acid, each of which is a separate storage unit wrapped in a polymer film to control the release rate of the drug. The tablets disintegrate rapidly after contact with the liquid, and the particles are dispersed on the huge surface of the gastrointestinal tract. The release of the drug is not affected by the $\mathrm{pH}$ value of the surrounding liquid, and is released at an almost constant rate of about $20 \mathrm{~h}^{[2]}$. The dosage form has a stable blood concentration, ACTS for more than 24 hours, and is completely absorbed after oral administration. Drug absorption occurs throughout the gastrointestinal tract, including the colon, with a bioavailability of $30 \%$ $40 \%{ }^{[3]}$. The treatment of metoprolol in liver metabolism can reduce the effect of catecholamines related to physical and psychological load, reduce heart rate, cardiac output and blood pressure, increase ejection fraction, and reduce the volume of end-systolic and 
end-diastolic left ventricle ${ }^{[4]}$. Trimetazidine belonging to another class of angina, cardiovascular drugs, absorb quickly, 2 hours to reach peak plasma concentration, by protecting cells in energy metabolism of ischemia or hypoxia and prevent a decrease in the level of ATP in the cell, so as to ensure the normal function of the ion pump and through the normal operation of membrane sodium/potassium flow, and maintain the stability of the intracellular environment ${ }^{[5-6]}$.

In summary, metoprolol succinic acid sustained release tablets combined with trimetazidine in the treatment of gastrointestinal tumors with angina pectoris can improve the efficacy of patients with angina pectoris, and the drug is safe, worthy of clinical use.

\section{References}

[1] Hao AY. Clinical efficacy observation of trimetazidine combined with rosuvastatin in the treatment of angina pectoris of coronary heart disease[J]. Journal of cardio-cerebrovascular diseases of integrated Chinese and western medicine, 2019, 17
(19): 3067-3069.

[2] Liu YJ, Song X. Effect of metoprolol on total myocardial ischemic load and heart rate variability in patients with angina pectoris due to coronary heart disease[J]. Journal of integrated Chinese and western cardio-cerebrovascular diseases, 2019, 17 (12): 1869-1871.

[3] Wei F, Bai MS. Efficacy of qisen capsule combined with metoprolol in the treatment of coronary heart disease angina pectoris (qi-deficiency and blood-stasis syndrome) $[\mathrm{J}]$. Chinese medical emergency, 2019, 28 (7): 1258-1261.

[4] Ruan JS, Peng L, Zheng B, et al. Efficacy analysis of metoprolol combined with safflower yellow pigment injection in the treatment of unstable angina pectoris of coronary heart disease[J]. Western medicine, 2019, 31 (10): 1601-1604.

[5] Mo JC, Yang NN, Wang ZM. Clinical effect observation of trimetazidine combined with tigrillo in the treatment of unstable angina with chronic heart failure $[\mathrm{J}]$. Chinese primary medicine, 2019, 26 (23): 2882-2885.

[6] Tang ML, Lu JQ, Yu MP, et al. Systematic evaluation and Meta analysis of efficacy and safety of wenxin granule combined with trimetazidine in the treatment of unstable angina $[\mathrm{J}]$. Journal of hunan university of traditional Chinese medicine, 2019, 39 (7): 898-906. 